\title{
A produção científica da classificação internacional de funcionalidade, incapacidade e saúde para crianças e jovens - CIF-CJ
}

\author{
Cristiane Paiva-Alves* \\ Aline Cirelli Coppede** \\ Maria Cristina Piumbato Innocentini Hayashi*** \\ Cláudia Maria Simóes Martinez****
}

\section{Resumo}

A CIF-CJ constitui-se numa ferramenta com abordagem biopsicossocial da funcionalidade humana e incapacidade específica para o período da infância permitindo uma linguagem comum entre os profissionais que lidam com crianças com incapacidades. Objetivo: descrever como se configura os estudos científicos que utilizam a CIF-CJ nas bases de dados nacionais e internacionais. Metodologia: pesquisa exploratória e descritiva com abordagem bibliométrica. As buscas foram realizadas nas bases de dados Bireme, Science Direct e Scopus; foi realizada leitura minuciosa dos estudos selecionados, preenchendo um protocolo com os principais dados de cada estudo; com o auxílio do Software VantagePoint ${ }^{\circledR}$, as análises dos dados produziram indicadores: ano de publicação; autoria; formação dos autores; periódicos; delineamento dos estudos; principais temas abordados. Resultados: crescimento de publicaçóes sobre o assunto nos últimos anos; a maioria dos estudos é de autoria coletiva; autores são da área de educação e saúde; o periódico que se destaca é o Disability and Rehabilitation; grande parte dos estudos descritivos tem como foco mapear escalas motoras e de desempenho infantil, programas de saúde e de educação ou patologias. Conclusão: os estudos revelam a importância do uso da CIF-CJ na prática clínica, porém não há estudos sobre sua viabilidade. Faltam instrumentos baseados nos parâmetros da CIF-CJ para avaliar o desenvolvimento infantil.

Palavras-chave: Classificaçáo internacional de funcionalidade, incapacidade e saúde para crianças e jovens; CIF-CJ; Produção científica.

\footnotetext{
* Professora doutora do Centro Universitário Claretiano de Batatais, Batatais, São Paulo, Brasil.

** Professora doutora da Universidade Estadual Paulista Júlio de Mesquita Filho, Marília, São Paulo, Brasil.

*** Professora doutora da Universidade Federal de São Carlos, São Carlos, São Paulo, Brasil.

**** Professora doutora da Universidade Federal de São Carlos, São Carlos, São Paulo, Brasil.
} 


\section{The scientific production of international classification of functioning disability and health for children and youth - ICF-CY}

\section{Abstract}

The ICF-CY constitutes a tool with biopsychosocial approach to human functioning and disability specific to the period of childhood allowing a common language among professionals who deal with children with disabilities. The aim of this study was to describe the configuration scientific studies using the ICF-CY in national and international databases. Methodology: exploratory and descriptive with bibliometric approach. The searches were conducted in the databases Bireme, Science Direct and Scopus; was made detailed reading of the selected studies, forming a protocol with key data from each study; with the assistance of VantagePoint Software ${ }^{\circledR}$, the analyzes of the data produced indicators: year of publication, authorship, training of authors, journals, study design, leading themes. Results: increase of publication on this subject in recent years, most studies are collective authorship; authors are from the area of education and health, the journal that stands out is the Disability and Rehabilitation; most descriptive studies with a focus on mapping scales motor performance and child health programs and education or pathologies. Conclusion: studies show the importance of using the ICF-CY in clinical practice, but there are no studies on its feasibility. Lack of instruments based on the ICF-CY parameters to assess child development.

Keywords: International classification of functioning; Disability and health for children and youth; ICF-CY; Scientific production.

\section{Introdução}

A CIF-CJ (Classificação Internacional de Funcionalidade Incapacidade e Saúde para Crianças e Jovens - WHO, 2007) é uma classificação biopsicossocial que considera a existência de um sistema complexo e inter-relacional composto por fatores que podem influenciar de forma positiva ou negativa o desenvolvimento de crianças e jovens, dependendo das interações que vão ocorrer entre os integrantes do sistema. A avaliação dos fatores biopsicossociais e suas possíveis interaçôes podem ser sistematizadas, a partir do seu uso, conforme mostra a Figura 1. 
Figura 1 - Interação entre os componentes da CIF. Fonte: OMS,2004

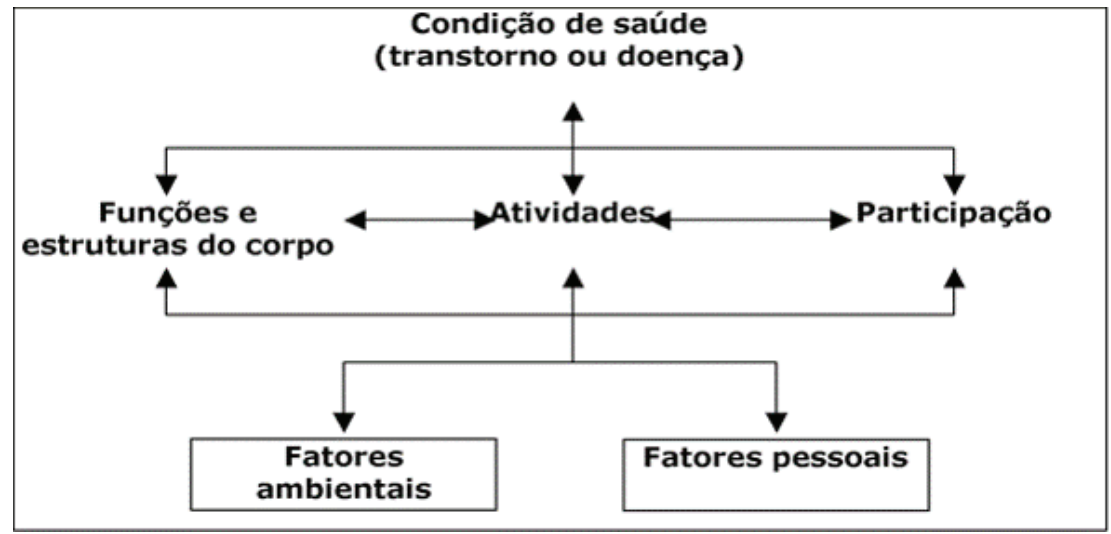

A CIF-CJ é uma versão derivada da CIF (Classificação Internacional de Funcionalidade, Incapacidade e Saúde) que contempla conteúdos específicos e detalhes adicionais, relevantes na infância e na adolescência. Ambas conceituam deficiência como produto da interação entre seus componentes: funçóes e estruturas do corpo, atividade, participação, fatores ambientais e pessoais (fatores contextuais) com o estado de saúde (WHO, 2007). Um exemplo desta interação se dá no contexto escolar, no qual a criança com déficits motores se insere, interagindo com o ambiente que pode facilitar ou dificultar na sua funcionalidade.

De acordo com Simeonsson et al. (2006), a CIF-CJ pode contribuir para a prática, política e pesquisa de muitas maneiras, tais como: a) fornecer uma estrutura para a prática interdisciplinar; b) traçar perfis da funcionalidade e rendimento da criança; c) esclarecer diagnósticos e comorbidades clínicas; d) fornecer, com base na funcionalidade, planejamento para tratamentos/intervençóes particularizados; e) ofertar códigos para identificar resultados de intervençôes; f) fornecer documentação sobre as mudanças ocorridas na funcionalidade; e g) esquematizar a documentação das variáveis da pesquisa.

Apesar de dar subsídio para a classificação do estado de saúde, a CIF-CJ não se constitui em um instrumento de avaliação, mas, serve como recurso que orienta $o$ planejamento e a aplicação em diversos contextos de avaliaçóes.

A maioria dos estudos desenvolvidos até o momento foca em efeitos físicos isolados ou aspectos psicossociais do desenvolvimento das crianças. Entretanto, estes estudos fornecem retrato limitado, centrando-se apenas em aspectos isolados da saúde. Essa realidade mostra que muitos estudos ainda precisam ser realizados para fundamentar a operacionalizaçẫo da CIF-CJ na construção de instrumentos para o desenvolvimento infantil.

Além da CIF-CJ fornecer parâmetros para a elaboração de um instrumento para avaliação motora de crianças em idade escolar, a produção científica sobre essa 
temática tem papel relevante, pois é através da literatura de referência da área que o campo científico da CIF-CJ nos mostrará os principais achados e quais lacunas ainda precisam ser preenchidas. Contudo, o estado da arte de um campo de conhecimento permite que profissionais aprimorem seus conhecimentos, conhecendo, avaliando e questionando as perspectivas e as tendências da área.

A produção científica é definida como atividades intelectuais intencionais que visam responder às atividades humanas, para compreender e transformar a realidade que nos rodeia. Significa realizar esforços para investigar, descobrir, conhecer algum fenômeno (VIANNA; ENSSLIN; GIFFHORN, 2011).

Com base nessas consideraçóes, foram delineadas as seguintes questóes de pesquisa do estudo: A CIF-CJ é utilizada como parâmetro para construção de instrumentos? Quais as tendências metodológicas, temáticas e perspectivas dos estudos sobre a CIF-CJ presentes nas bases de dados nacionais e internacionais? Para responder essas questóes formulou-se o objetivo geral da pesquisa: descrever e analisar como se configuram os estudos sobre a CIF-CJ presentes nas bases de dados nacionais e internacionais.

\section{Metodologia}

A pesquisa é de caráter exploratório e descritivo, com abordagem da bibliometria. Esta é uma avaliação objetiva da produção científica e permite identificar o comportamento da literatura e sua evoluçáo em contexto e época determinados (PIZZANI; BELLO; HAYASHI, 2010). Através da bibliometria é possível construir indicadores destinados a avaliar a produção científica de indivíduos, áreas de conhecimento e países, sendo estes indicadores empregados na avaliaçáo de pesquisadores e áreas de conhecimento. (SILVA; HAYASHI; HAYASHI, 2011).

Para identificação dos estudos, foram consultadas as seguintes bases de dados: BVS-BIREME (Biblioteca Virtual de Saúde do Centro Latino-Americano e do Caribe de Informação em Ciências da Saúde, (disponível em http://www.bireme.br); ScienceDirect (disponível em http://www.sciencedirect.com), e Scopus (disponível em http:// www.scopus.com). As buscas realizaram-se em outubro de 2012. Utilizou-se um único termo de busca para pesquisa em todas as bases: "ICF-CY" (sigla de International Classification of Functioning, Disability and Health for Children and Youth).

Para ser incluído na pesquisa, o trabalho deveria ter sido publicado na forma de artigo e estar disponível para acesso no texto completo. Foram excluídos os trabalhos que a busca retornou em que não havia o escopo da CIF-CJ da maneira esperada, ou seja, artigos que falavam da CIF em geral e apenas citavam a existência da CIF-CJ, mas não a utilizavam no estudo; foram excluídos também teses e dissertaçóes, artigos nos quais não foram possíveis acessar o texto na íntegra e artigos duplicados.

O período de busca não foi delimitado, pois o foco da pesquisa consistiu em mapear a evolução da produção científica sobre esse tema. Assim, foram considerados desde o registro mais antigo até o mais atual. Os resultados encontrados foram tabulados. Dois revisores, de forma independente, realizaram a leitura criteriosa dos títulos e dos resumos/abstracts dos estudos identificados na busca. Os que atende- 
ram aos critérios de inclusão foram selecionados. Finalizada a seleção, os revisores se reuniram para entrar em consenso sobre a inclusão ou exclusão dos artigos. Caso houvesse desacordo, um terceiro revisor seria solicitado para resolver as diferenças; no entanto, isso não foi necessário. Após essa etapa, os estudos selecionados foram lidos na íntegra e analisaram-se os seguintes aspectos: idioma; país e área de formação do autor (nos casos de autoria coletiva, considerou-se o primeiro autor); ano de publicação; periódico, delineamento dos estudos; temática mais abordada.

Foram identificados 263 estudos na primeira abordagem de busca. Em seguida, a partir dos critérios de pré-seleção (análise de títulos e resumos), foram selecionados 64, sendo que, desses, foram descartados 25 localizados na base Scopus, pois estavam duplicados na BVS (21) e ScienceDirect (4) e mais 1 duplicado na base ScienceDirect. Ao final, foram selecionados 38 estudos que compuseram o corpus analisado, conforme pode ser observado na Tabela 1 .

Tabela 1 - Descrição da coleta de dados nas bases científicas.

\begin{tabular}{l|l|l|l|l}
\hline Base de dados & $\begin{array}{l}\text { Artigos } \\
\text { disponíveis }\end{array}$ & $\begin{array}{l}\text { Artigos pré- } \\
\text { selecionados }\end{array}$ & Duplicados & $\begin{array}{l}\text { Artigos } \\
\text { selecionados }\end{array}$ \\
\hline BIREME & 65 & 23 & - & 23 \\
\hline SCIENCE DIRECT & 90 Refinado & 6 & $1^{*}$ & 5 \\
\hline SCOPUS & 108 & 35 & $\begin{array}{l}21^{*} \\
4^{* *}\end{array}$ & 10 \\
\hline TOTAL & 263 & 64 & 26 & 38 \\
\hline
\end{tabular}

Fonte: $w w w$.bireme.br; data da coleta: Out/2012; www.sciencedirect.com data da coleta: Out/2012; www.scopus.com data da coleta: Out/2012. Legenda: Refinado - Tipo - Journal; Ano - 2006 a 2012. * duplicado com a BVS; ${ }^{* *}$ duplicado com a SicenceDirect;

Os dados foram registrados em um protocolo de coleta de dados bibliométricos elaborado por Hayashi et al (2011); em seguida foram transpostos para o software VantagePoint ${ }^{\circ}$ que permite realizar as análises bibliométricas. Para melhor visibilidade dos resultados obtidos foi utilizada a planilha MS Excel que possibilita a criação de tabelas e gráficos.

Os aspectos éticos da pesquisa (os dados coletados e a identificação dos seus autores são de domínio público, disponíveis em bases de dados online) entende-se que a exigência de submissão e aprovação do Comitê de Ética seja dispensável. No entanto, foram observados os aspectos éticos da pesquisa científica, em relação à honestidade e precisão em relaçáo aos dados coletados, o que implicou em respeito à autoria científica e fidedignidade as ideias dos autores dos textos analisados.

\section{Resultados}

Dentre os artigos selecionados, observa-se que a publicação mais antiga foi em 2006, apresentando um número crescente das publicações ao longo dos anos. 
Ao analisar a produção científica ao longo dos anos, foi possível observar a evolução e crescimento deste campo de conhecimento. A figura 2 apresenta a distribuição das publicaçóes ao longo dos anos.

Figura 2 - Distribuição das publicaçôes ao longo do tempo.

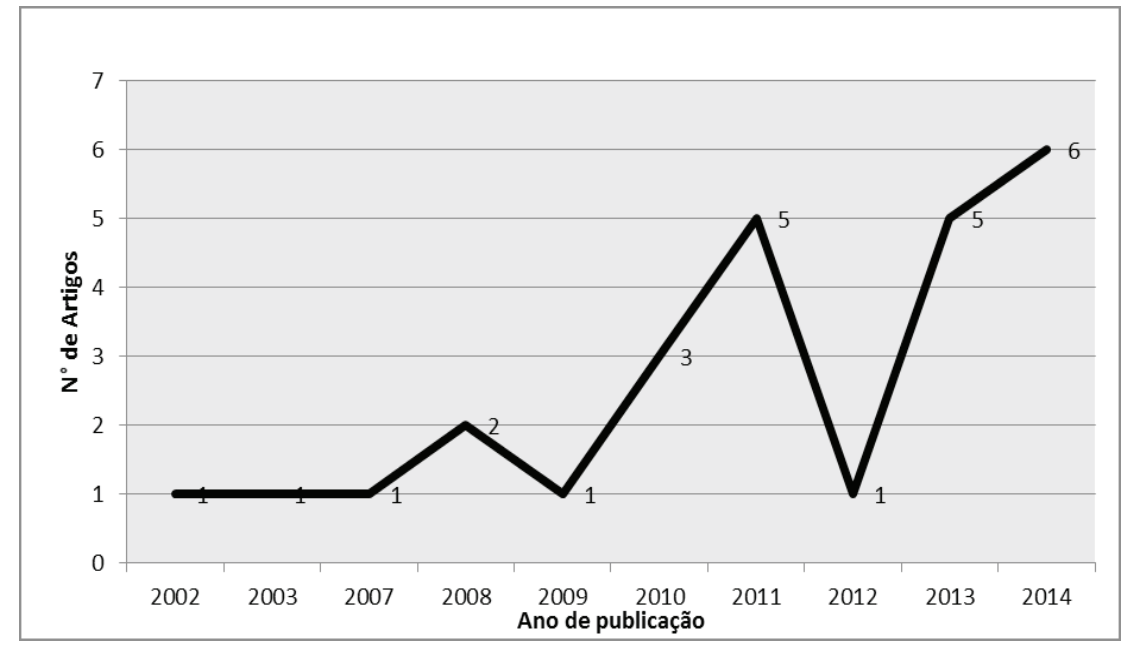

Observa-se que a produção científica sobre a CIF-CJ tem crescido ao longo dos anos, com um aumento significativo em 2012. Não foi incluído na figura os 2 artigos de 2013, porque na época em que a coleta foi feita os artigos de 2013 ainda não estavam publicados, sendo estes dois no modelo disponíveis no modo "in press" na base de dados. De acordo com Laudelino, Navarro e Beuren (2010), estudar a evolução das pesquisas ao longo do tempo é um fator importante, pois a contagem das publicaçôes, como indicador bibliométrico, é um instrumento de análise que se presta a difundir achados científicos e colabora com a política de revisão científica destinada a medir e avaliar as contribuiçóes à ciência, demonstrando o fortalecimento ou o aumento do interesse de um determinado conhecimento pela comunidade científica.

Assim, o campo da CIF-CJ tem mostrado um crescimento ao longo dos anos, podendo-se inferir que o interesse por essa temática na comunidade científica tem aumentado desde a sua publicação em 2007.

Visando identificar se há colaboração científica nos artigos selecionados, apresentamos a tabela 2, com a distribuição dos artigos de acordo com a autoria única e coautorias. 
Tabela 2 - Indicadores de autoria.

\begin{tabular}{|l|l|l|}
\hline Autoria & Frequência Absoluta & Frequência Relativa (\%) \\
\hline 1 & 6 & 16 \\
\hline 2 & 4 & 11 \\
\hline 3 & 7 & 18 \\
\hline 4 & 10 & 26 \\
\hline 5 & 3 & 7 \\
\hline 7 & 2 & 5 \\
\hline 8 & 4 & 11 \\
\hline 10 & 1 & 3 \\
\hline Total & 1 & 3 \\
\hline & 38 & 100 \\
\hline
\end{tabular}

Apenas $6(16 \%)$ estudos foram escritos em autoria individual, sendo grande parte escrita com mais de 2 autores, destaque para os estudos com 4 autores que representam $26 \%$ do total de artigos.

Quando se analisam os indicadores de autoria e produtividade dos autores, os resultados apontam que para a elaboração de artigos científicos há certa preferência por parte da comunidade acadêmica em formar redes de colaboração, ou seja, publicar em parceria com outros autores ao invés de publicar sozinho. A pesquisa em colaboração parece ser mais visível do que a pesquisa individual e os trabalhos mais citados em uma determinada área do conhecimento são frequentemente escritos em colaboração (SAES, 2000).

Além disso, a frequência relativa do número de trabalhos escritos em colaboração entre grupos é proporcional ao grau de cooperação científica do grupo e fornece um índice do grau de cooperação (SAES, 2000). Atualmente, o esforço colaborativo entre vários autores está representado nas publicaçóes, em parte pela multidisciplinaridade de diversas áreas ou pelo suporte financeiro recebido em determinados estudos.

Donato e Oliveira (2006) chamam a atenção para dois outros pontos importantes quando se trata do aumento do número de autores por documento. Esses autores se referem à Sindrome POP - Publish or Perish, cujo lema é: "publique ou pereça", que força o pesquisador a publicar cada vez mais trabalhos científicos para alcançar um posicionamento profissional favorável e também porque as investigaçóes estão cada vez mais complexas, especializadas e custosas, justificando maior colaboração.

Assim, os pesquisadores buscam desenvolver o maior número de projetos e atividades que resultem em produtos quantificáveis representados pelos indicadores de resultados que vão fornecer elementos para registro, contabilização e avaliação do conjunto da produção entre os cientistas (PIZZANI; SILVA; HAYASHI, 2008).

Outro indicador observado é o de produtividade dos autores, os 38 estudos selecionados foram escritos por um total de 122 autores. Desse total 13 autores se 
destacam com mais de uma publicaçáo sobre a temática, a tabela 3 apresenta a produtividade desses autores.

Tabela 3 - Indicadores de produtividade dos autores.

\begin{tabular}{c|c|c|}
\hline Autor & FA & FR (\%) \\
\hline Mats Granlund & 7 & 13 \\
\hline Rune J. Simeonsson & 5 & 1111 \\
\hline Eva Bjorck-Akesson & 4 & 11 \\
\hline Margareta Adolfsson & 4 & 8 \\
\hline Mia Pless & 4 & 8 \\
\hline Matilde Leonardi & 3 & 5 \\
\hline Nina Klang Ibragimova & 3 & 5 \\
\hline Andrea Martinuzzi & 3 & 5 \\
\hline Alberto Raggi & 2 & 5 \\
\hline Ana Isabel Pinto & 2 & 5 \\
\hline Donald J. Lollar & 2 & 5 \\
\hline Susana Castro & 2 & 5 \\
\hline Wendy Coster & 2 & 19 \\
\hline
\end{tabular}

Com relação à formação dos autores a figura 3 apresenta os resultados. Observa-se uma variedade de áreas que pesquisam esse assunto, porém todas elas inserem-se nos contextos da saúde e educação, que entendem o indivíduo como um ser biopsicossocial, justificando assim a diversidade dos campos. Além disso, em termos de aplicabilidade, a CIF já foi amplamente utilizada nos setores da Saúde, Educação, Seguridade Social, Economia e Desenvolvimento, entre outros (WHO, 2007).

Figura 3 - Indicadores de formação dos autores.

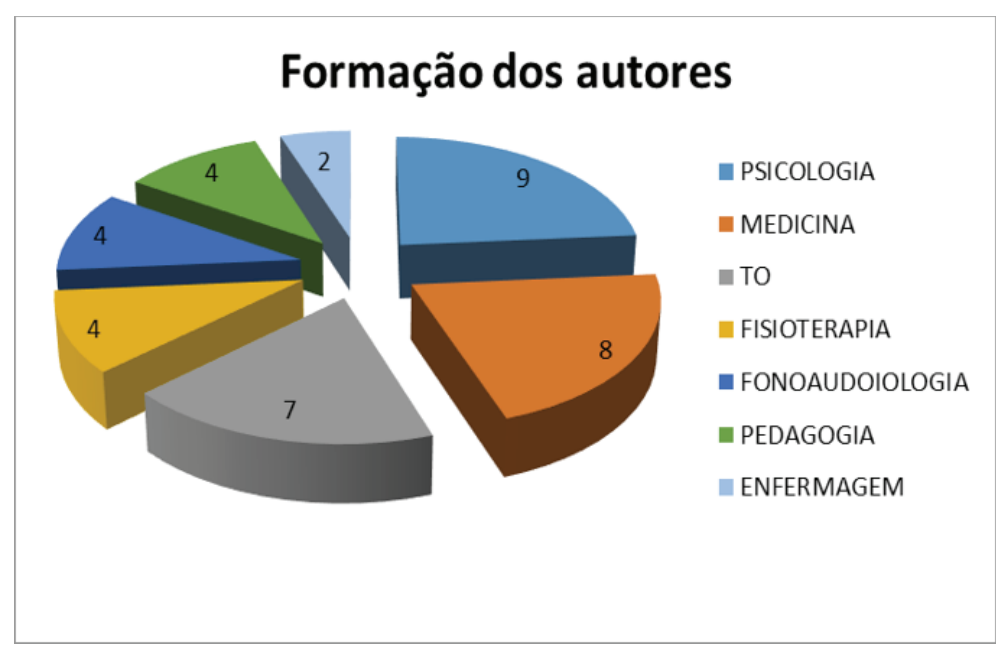


Considerando o perfil dos artigos selecionados, os dados revelam que o Inglês é o idioma predominante nos estudos sobre esta temática, sendo que todos (100\%) os artigos foram publicados nessa língua. Porém quando observamos o país de origem dos autores, observamos que a Suécia lidera como o país que mais tem publicado sobre a CIF-CJ. A figura 4 mostra a distribuição dos estudos de acordo com o país.

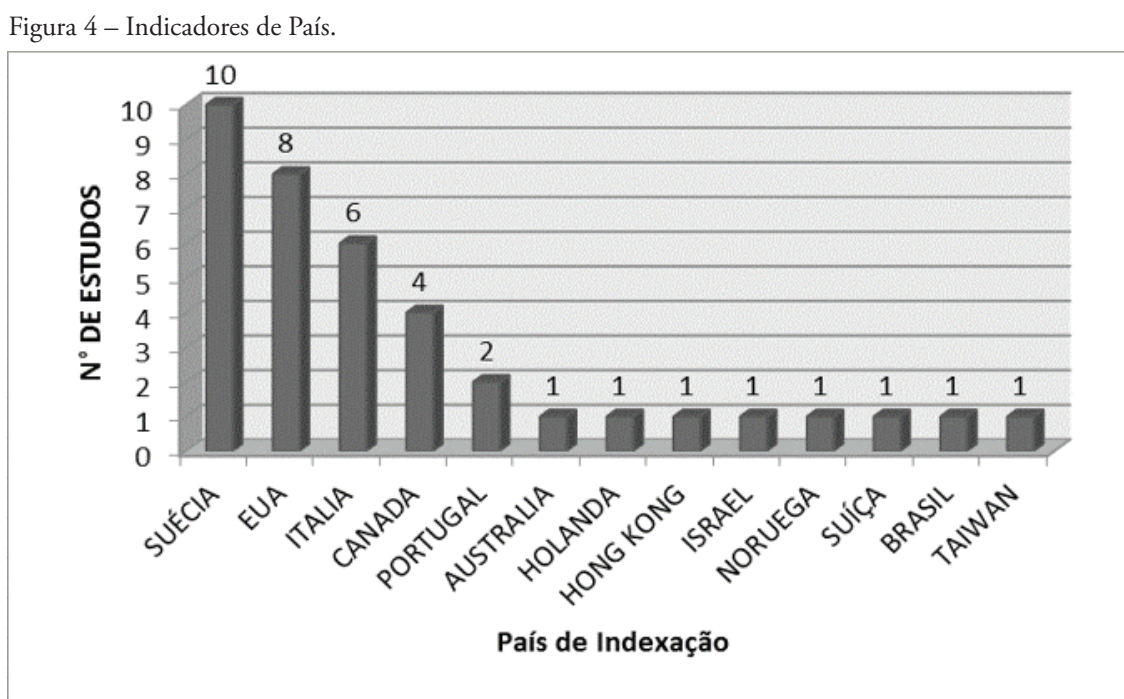

Para uma melhor identificação dos periódicos nos quais os estudos foram publicados, elaboramos a tabela 4 a seguir.

Tabela 4 - Indicadores dos periódicos.

\begin{tabular}{l|c|c|}
\hline PERIÓDICO & FA & FR (\%) \\
\hline $\begin{array}{l}\text { Disability and } \\
\text { Rehabilitation }\end{array}$ & 11 & 29 \\
\hline $\begin{array}{l}\text { Augmentative } \\
\text { and Alternative } \\
\text { Communication }\end{array}$ & 3 & 8 \\
\hline $\begin{array}{l}\text { Physical \& Occupational } \\
\text { Therapy in Pediatrics }\end{array}$ & 3 & 5 \\
\hline $\begin{array}{l}\text { Developmental Medicine } \\
\text { \& Child Neurology }\end{array}$ & 2 & 5 \\
\hline $\begin{array}{l}\text { Developmental } \\
\text { Neurorehabilitation }\end{array}$ & 2 & 5 \\
\hline $\begin{array}{l}\text { Journal of } \\
\text { Rehabilitation Medicine }\end{array}$ & 2 & 3 \\
\hline $\begin{array}{l}\text { Advances in Speech- } \\
\text { Language Pathology }\end{array}$ & 1 & \multicolumn{2}{|c|}{} \\
\hline
\end{tabular}


Continuação da Tabela 4.

\begin{tabular}{|c|c|c|}
\hline $\begin{array}{l}\text { Americam Speech } \\
\text { Language Hearing } \\
\text { Association - ASHA }\end{array}$ & 1 & 3 \\
\hline $\begin{array}{l}\text { Archives of } \\
\text { Physical Medicine } \\
\text { and Rehabilitation }\end{array}$ & 1 & 3 \\
\hline Clinical Rehabilitation & 1 & 3 \\
\hline $\begin{array}{l}\text { European Early } \\
\text { Childhood Education } \\
\text { Research Journal }\end{array}$ & 1 & 3 \\
\hline $\begin{array}{l}\text { Health and Quality of } \\
\text { Life Outcomes }\end{array}$ & 1 & 3 \\
\hline $\begin{array}{l}\text { International Journal of } \\
\text { Social Welfare }\end{array}$ & 1 & 3 \\
\hline $\begin{array}{l}\text { Journal of Child } \\
\text { Neurology }\end{array}$ & 1 & 3 \\
\hline $\begin{array}{l}\text { Journal of Intellectual \& } \\
\text { Developmental Disability }\end{array}$ & 1 & 3 \\
\hline $\begin{array}{l}\text { Journal of Policy and } \\
\text { Practice in Intellectual } \\
\text { Disabilities }\end{array}$ & 1 & 3 \\
\hline $\begin{array}{l}\text { Journal of the Formosan } \\
\text { Medical Association }\end{array}$ & 1 & 3 \\
\hline Maturitas & 1 & 3 \\
\hline $\begin{array}{l}\text { Research in } \\
\text { Developmental } \\
\text { Disabilities }\end{array}$ & 1 & 3 \\
\hline $\begin{array}{l}\text { Scandinavian Journal of } \\
\text { Public Health }\end{array}$ & 1 & 3 \\
\hline $\begin{array}{l}\text { The British Journal } \\
\text { of Developmental } \\
\text { Disabilities }\end{array}$ & 1 & 3 \\
\hline TOTAL & 38 & 100 \\
\hline
\end{tabular}

De acordo com Pizzani, Silva e Hayashi, 2008, as pesquisas precisam ser publicadas para que seus resultados sejam conhecidos no meio científico.

As revistas científicas representam importantes meios de disseminação desses resultados, pois além de serem maneiras dinâmicas e rápidas de divulgar o conhecimento produzido, é nelas que a comunidade pode avaliar a justa medida da pesquisa, pois o pesquisador precisa dizer o essencial, e com concisão, já que as páginas são limitadas (AMORIM et al., 2007; LAVILLE; DIONNE, 1999). 
Do ponto de vista da análise da produção científica, as revistas fornecem importantes indicadores bibliométricos como o fator de impacto, índice de imediatez e influência, os quais permitem, por exemplo, avaliar a frequência com que um artigo de uma determinada revista é citado num determinado ano; medir com que rapidez um determinado artigo é citado após sua publicação; ou avaliar a repercussão que exercem as citaçóes recebidas por uma revista. Esta repercussão depende do mérito científico do periódico, de sua ampla ou restrita circulaçáo, de sua disponibilidade, de seu grau de especialização, seu país de origem e idioma.

Ao analisar a produção científica dos estudos sobre a CIF-CJ, observou-se que dos 38 artigos selecionados encontramos 3 tipos distintos de delineamentos: estudos exploratórios (11), descritivos (26) e experimentais (1).

Segundo Gil (2002) o delineamento da pesquisa refere-se ao planejamento da mesma em sua dimensão mais ampla, ou seja, o pesquisador deve estabelecer os meios técnicos da investigaçáo prevendo-se os instrumentos e procedimentos necessários que utilizará para a coleta de dados.

Desta forma a pesquisa pode ser classificada em:

Pesquisas Exploratórias: têm como objetivo o aprimoramento de ideias, proporcionar maior familiaridade com o problema, com vistas a torná-lo mais explícito. $\mathrm{Na}$ maioria das vezes a pesquisa exploratória envolve pesquisa bibliográfica e estudo de caso (GIL, 2002);

Pesquisas Descritivas: têm como objetivo a descrição das características de uma determinada população, fenômeno ou estabelecer relações entre variáveis. Como exemplo, pesquisas que estudam as características de um grupo: distribuição por idade, sexo, escolaridade, estado de saúde, etc.; estudam o nível de atendimento dos órgãos públicos de uma comunidade, suas condiçôes de habitação, nível de criminalidade, etc.; levantam as opiniōes, atitudes e crenças de uma população; descobrem a associação entre variáveis, como nas pesquisas eleitorais que indicam a relação entre preferência partidária e escolaridade. Geralmente as pesquisas descritivas assumem a forma de levantamento (GIL, 2002);

Pesquisas Experimentais ou Explicativas: têm como objetivo identificar os fatores que determinam ou que contribuem para a ocorrência dos fenômenos. Uma pesquisa experimental pode ser a continuação de outra descritiva, pois explicar um fenômeno exige sua descrição detalhada (GIL, 2002).

A figura 5 representa a classificação dos delineamentos dos estudos. 
Figura 5 - Indicadores de delineamento dos estudos.

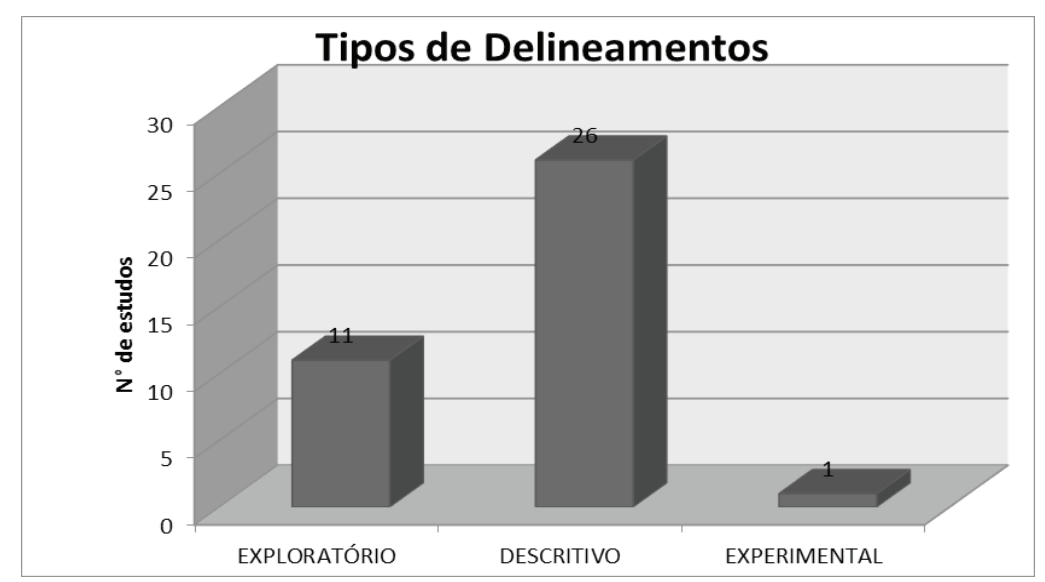

Além disso, foi realizada uma classificação dos artigos, de acordo com os objetivos dos estudos, identificando a finalidade do uso da CIF-CJ na pesquisa em questão. Elaboramos a figura 6 para melhor visualização dos dados.

Figura 6 - Classificação dos estudos sobre a CIF-CJ.

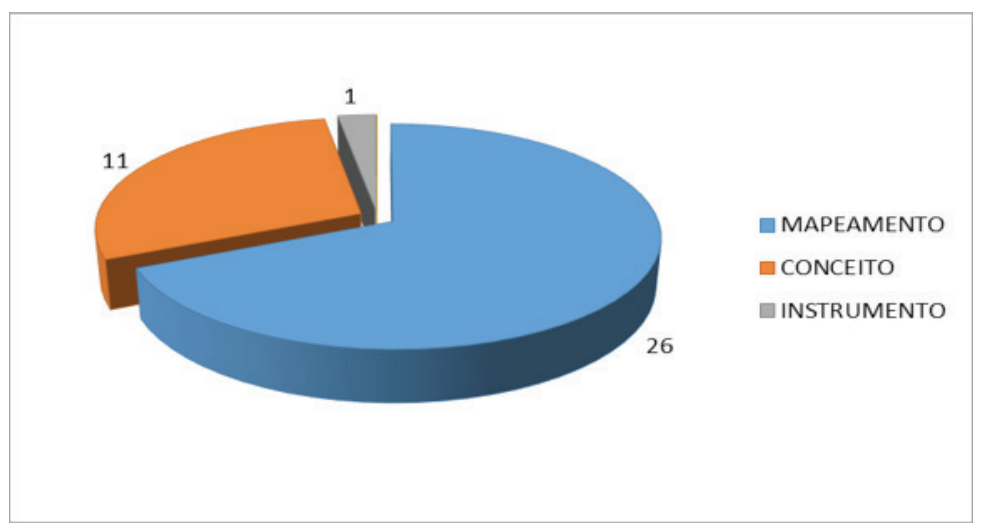

Classificamos como mapeamento todos os estudos que utilizaram a CIF-CJ para mapear instrumentos já existentes, ou seja, buscar entre os domínios da CIF-CJ os itens dos instrumentos elencados. E também os estudos que utilizaram a CIF-CJ como protocolo de avaliação ou guia para classificação de serviços, estratégias de intervenção ou pesquisas. Em vista disso, grande parte dos estudos selecionados foi classificada como estudo de mapeamento (67\%). Dentre eles, 16 estudos utilizaram as "linking rules", descritas por Cieza et al. (2005) para mapear ou diagnosticar a funcionalidade e incapacidade dos sujeitos. As "linking rules" são regras utilizadas para ligar a CIF com outros instrumentos ou testes, codificando os itens de escalas ou testes com os seus códigos. 
Do total de artigos que utilizaram as "linking rules", 13 (81\%) codificaram testes motores e de desempenho infantil baseados nos códigos da CIF-CJ, identificando quantos itens avaliavam estruturas e funçóes do corpo, quantos itens quantificavam atividades e participação, etc. Verificando assim quais domínios da CIF-CJ estavam presentes nos testes, na tentativa de verificar a utilizaçáo da Classificação na prática com crianças com deficiência.

O estudo de Castro, S., Pinto, A., \& Simeonsson, R. J. (2012) utilizou a CIFCJ para mapear 33 programas de educaçáo especial para crianças com autismo. As autoras identificaram que a maioria dos domínios abordados nos programas relaciona-se com atividades e participação, nenhum programa incluiu os fatores ambientais em algum momento do planejamento do programa.

O estudo de Ståhl, Y., Granlund, M., Gäre-Andersson, B., \& Enskär, K. (2011) ligaram os dados de saúde de todas as crianças da Suécia nos serviços de saúde e educação com os códigos da CIF-CJ. Identificaram que em alta proporção os dados referem-se a funçôes e estruturas do corpo e atividades e participação, porém, fatores ambientais aparecem em baixíssima proporção. O estudo de Andrade et al (2012), identificaram a frequência dos itens de funcionalidade e incapacidade que estavam presentes nas avaliaçóes multiprofissionais de crianças com paralisia cerebral de acordo com a CIF-CJ, encontrando uma variedade nas proporçóes das categorias, funçóes e estruturas do corpo, atividades e fatores ambientais, sugerindo estabelecer um consenso interdisciplinar para registro das informaçóes.

Outros 10 artigos não utilizaram-se das "linking rules" para codificar nenhum teste ou serviço, porém realizaram o mapeamento de alguns serviços ou patologias, utilizando a CIF-CJ como um protocolo de avaliaçáo, selecionando os seus domínios.

Gilboa e colaboradores (2010) utilizaram a CIF-CJ para mapear as características de crianças com neurofibrose, e o impacto da doença em suas atividades diárias; De modo semelhante Trabacca et al (2012) utilizaram a CIF-CJ para mapear e planejar a intervenção de uma criança com paralisia cerebral; Meucci et al (2009) fizeram o mesmo com crianças com síndrome de Tourette, e Bonani et al. (2009), de modo semelhante com crianças com Síndrome de Angelmans.

No estudo de De Polo et al (2009) usaram a CIF-CJ na elaboração de um protocolo para inclusão de crianças com deficiência na escola. Usando os domínios da CIF-CJ para realizar diagnóstico funcional, elaborar um plano de intervenção e avaliação individual do aluno incluído.

Outros autores como Hwang e colaboradores (2012), seguindo a mesma metodologia utilizaram a CIF-CJ como instrumento para avaliação e acompanhamento do desenvolvimento infantil desde o nascimento até 2 anos de idade, identificando as influencias do ambiente familiar.

Assim, Björck-Åkesson et al. (2010), mapearam um serviço de intervenção precoce objetivando uma linguagem única entre os profissionais que compóem a equipe de atendimento. Já, Hollenweger, J. (2010) qualificou o funcionamento de um sistema educacional para crianças com deficiência com base na Classificação. 
Rowland e colaboradores (2012) fizeram o mesmo para um programa de comunicação alternativa na escola com crianças com problemas de fala. Contudo, Martinuzzi et al. (2010) também, utilizaram a CIF-CJ para classificar um serviço hospitalar pediátrico na ala de neurologia;

Todos os estudos de mapeamento encontrados usam a CIF-CJ para identificar os domínios presentes em patologias, serviços de saúde e de educação. E o principal resultado encontrado é que a CIF-CJ se constitui como um importante e útil instrumento para prática, permite uma linguagem universal e detalhada num contexto biopsicossocial, levando em consideração a influência de diversos fatores físicos, psíquicos e ambientais.

Foram considerados estudos conceituais, aqueles que utilizaram a CIF-CJ para explicar, comparar ou refletir sobre os componentes e suas definiçóes. São os estudos que trazem alguma reflexáo sobre a importância do novo paradigma trazido pela CIF, dentro de suas áreas de interesse.

Dos 38 artigos selecionados 13 se debruçaram sobre as questóes teóricas da classificação. $\mathrm{O}$ primeiro estudo que se relaciona aos conceitos foi realizado antes de sua publicação, com a utilização da versão beta da classificação, McLeod (2006), apresentou um estudo de caso de um menino de 7 anos com fala ininteligível e considerou os aspectos do seu estado de saúde de acordo com os componentes de função do corpo, estrutura corporal, atividade e participação, os fatores ambientais e fatores pessoais, identificando quais domínios haviam sido afetados por seu comprometimento da fala. Expondo uma tentativa preliminar para determinar as dimensôes salientes e os códigos da CIF-CJ (beta draft) pertinente ao uso por fonoaudiólogos que trabalham com crianças com fala ininteligível.

Outros estudos apresentam a estrutura da CIF-CJ e discutem a importância de sua terminologia para a comunicação interdisciplinar, de seu modelo e da necessidade de criaçáo de instrumentos que viabilizem sua aplicação nos diversos campos da infância (CRAMM et al. 2012; DIJKERS, 2010; SIMEONSSON; 2009). Em seu artigo Hoogsteen \& Woodgate (2010), descrevem as tendências do uso da CIF-CJ e citam que a terapia ocupacional pode colaborar para a conscientizaçáo sobre a importância do uso da classificaçáo, no sentido de clarificar a base de conhecimento da mesma, pois, acreditam que a CIF-CJ, assim como a terapia ocupacional, apresenta a proposta de mudar os padróes de práticas profissionais.

Pless et al. (2009) e Simeonsson et al. (2012) tratam dos conceitos da CIF-CJ aplicados a crianças com deficiência auditiva. Já, Bendixen et al. (2012) os relaciona a Distrofia de Duchene e as implicaçôes para avaliação com o uso da classificação na descrição de todos os componentes da vida de uma criança com Duchene, discutindo a sua importância para ajudar na promoção de sua funcionalidade e nos benefícios que essa avaliaçáo traria tanto para os pais, como para as crianças.

Seguindo a mesma linha de raciocínio, outros autores discutem o modelo da CIF-CJ e sua aplicação para crianças com Paralisia Cerebral, elencando quais componentes seriam importantes para a avaliação das mesmas (BRAENDVIK et al. 2010). 
Dois estudos começam a avançar do campo teórico para o prático, ou seja, a operacionalização da CIF-CJ no treinamento de profissionais e na criação de um instrumento, respectivamente. O de Self et al. (2011) explora elementos chaves e mecanismos de colaboração interprofissional em intervenção precoce, visando a preparaçáo de profissionais para a adequaçáo ao modelo de atuação proposto pela CIFCJ e sua utilização na saúde e educação. E o de Gleason \& Coster (2012) discute os conceitos da classificação e propóe, com base em fundamentação teórica, uma seleção de componentes para criação de um instrumento para avaliação de funcionalidade de crianças. $\mathrm{O}$ que parece ser a primeira etapa da construção de um instrumento.

No entanto, na categoria "instrumentos", ou seja, estudos que construíram um instrumento com o uso da CIF-CJ, identificamos apenas um artigo: "Ibragimova, Granlund, Bjorck-Akesson. Field trial of ICF version for children and youth (ICFCY) in Sweden logical coherence, developmental issues and clinical use (2009), que utilizou questionários da CIF-CJ como um instrumento de avaliação na prática clínica. Este estudo teve como objetivo investigar a viabilidade da CIF-CJ em questionários de avaliação. Para tanto, foram selecionados 139 profissionais que trabalhavam com intervenção em crianças com deficiência, para que utilizassem os questionários de avaliação, denominados ICF-CY Questionnaire. Os profissionais consideraram que o ICF-CY Questionnaire é uma ferramenta viável na avaliação e intervenção. No entanto, ainda existem várias questôes que devem ser desenvolvidas e discutidas para facilitar a implementação da CIF- CJ na prática clínica.

Nota-se que a maioria dos artigos trata a CIF-CJ de maneira conceitual ou fazem uso da classificação em conjunto com outros instrumentos para mapeamento e diagnósticos.

Observa-se, de acordo com os dados, que em questão da operacionalização da classificação houve um avanço pouco significativo, apenas um artigo se propôs a realizá-lo, e chegou-se a conclusão, a partir de análise realizada por profissionais, que apesar da CIF-CJ ser importante para a prática clínica, relatou-se a dificuldade do uso do questionário, bem como sua qualificação segundo a CIF. Este dado aponta para uma lacuna do conhecimento na operacionalização da CIF-CJ para a construçấo de instrumentos de avaliação.

As pesquisas revelam a importância do modelo proposto pela classificação, mas relatam dificuldades na aplicação prática para avaliação. Diante disso, a construção de instrumentos de avaliação baseados nos parâmetros biopsicossociais da CIF-CJ torna-se essenciais para detecção precoce de problemas no desenvolvimento infantil, específicos para as áreas física, social e psicológica.

\section{Considerações finais}

A análise bibliométrica dos artigos sobre a CIF-CJ revelou que essa produção científica tem crescido ao longo dos anos, sendo que elas foram publicadas em periódicos renomados de alta qualidade e rigor científico. Os estudos são todos em inglês, mas com destaque para a Suécia, que se mostra como país pioneiro nas publicaçóes sobre o assunto. A maioria dos artigos é de autoria coletiva, das áreas de educaçáo e 
saúde, com delineamentos descritivos ou exploratórios. Grande parte dos trabalhos tratam da CIF-CJ em conjunto com outros instrumentos para mapeamento e/ou diagnósticos.

Conclui-se a importância do uso da CIF-CJ na prática clínica, mas ainda há uma escassez de pesquisas sobre a viabilidade dela na prática. Apesar de alguns estudos compararem sua classificação com outros instrumentos, e de indicarem dificuldades na sua aplicaçáo, não há instrumentos baseados nos parâmetros da CIF-CJ para avaliar o desenvolvimento infantil e suas peculiaridades. Essa é uma das lacunas que o estudo bibliométrico permitiu verificar, além de fornecer subsídios para elaboração e divulgação de novas pesquisas nessa área.

\section{Referências}

AMORIM, K. P. C.; et al. A construção do saber em Odontologia: a produção científica de três periódicos brasileiros de 1990 a 2004. Interface - Comunicaçáo, Saúde, Educaçáo, v.11, n.21, p.9-23, 2007.

BENDIXEN, R. M.; SENESAC, C.; LOTT, D. J.; VANDENBORNE, K. Participation and quality of life in children with Duchenne muscular dystrophy using the International Classification of Functioning, Disability, and Health. Health and quality of life outcomes, v. 10, p. 43, 2012.

BJÖRCK-ÅKESSON, E.; et al. The International Classification of Functioning, Disability and Health and the version for children and youth as a tool in child habilitation/early childhood intervention--feasibility and usefulness as a common language and frame of reference for practice. Disability and rehabilitation, v. 32, Sup. 1, p. S125-38, 2010. Disponível em: <http://www.ncbi.nlm.nih.gov/pubmed/20843264>. Acesso em: 12 fev. 2013.

BONANNI, P; et al. Functioning and disability in patients with Angelman syndrome: utility of the International Classification of functioning disability and health, children and youth adaptation framework. Disability and rehabilitation. v. 31, 2009.

BRAENDVIK, S. M.; ELVRUM, A.-K. G.; VEREIJKEN, B.; ROELEVELD, K. Relationship between neuromuscular body functions and upper extremity activity in children with cerebral palsy. Developmental medicine and child neurology, v. 52, n. 2, p. 29-34, 2010.

CIEZA , A.; et al. ICF linking rules: an update based on lessons learned. Journal of Rehabilitation Medicine, v.37, p.212-218, 2005.

DE POLO, G.; PRADAL, M.; BORTOLOT, S.; BUFFONI, M.; MARTINUZZI, A. Children with disability at school: the application of ICF-CY in the Veneto region. Disability and rehabilitation, v. 31, n.1, p. S67-73, 2009.

DONATO, H. M.; OLIVEIRA, C. F. Patologia mamária: avaliação da atividade científica nacional através de indicadores bibliométricos. Acta Médica Portuguesa, v.19, p.225-234, 2006.

FLORIAN, L.; et al. Cross-cultural perspectives on the classification of children with disabilities: Part I. Issues in the classification of children with disabilities. Journal of Special Education, v. 40, n. 1, p. 3-45, 2006.

GIL, A. C. Como elaborar projetos de pesquisa. 4ed. São Paulo: Atlas, 2002.

GILBOA, Y.; ROSENBLUM, S.; FATTAL-VALEVSKI, A.; JOSMAN, N. Application of the International Classification of Functioning, Disability and Health in children with neurofibromatosis type 1: a review. Developmental medicine and child neurology, v. 52, n. 7, p. 612-9, 2010.

GLEASON, K.; COSTER, W. An ICF-CY-based content analysis of the Vineland Adaptive Behavior Scales-II. Journal of intellectual \& developmental disability, v. 37, n. 4, p. 285-293, 2012.

HAYASHI, M. C. P. I.; HAYASHI, C. R. M.; MARTINEZ, C. M. S. Estudos sobre jovens e juventudes: diferentes percursos refletidos na produçáo científica brasileira. Educaçáo, Sociedade \& Culturas, Porto, v.27, p.131-154, 2008.

HAYASHI, M. C. P. I. et al. Protocolo para coleta de dados bibliométricos em bases de dados. Mimeo. 2011. 
HOLLENWEGER, J. MHADIE's matrix to analyse the functioning of education systems. Disability and rehabilitation, v. 32, s. 1, p. S116-24, 2010. Disponível em: <http://www.ncbi.nlm.nih.gov/pubmed/20874661>. Acesso em: 4 abr. 2012.

HOOGSTEEN, L.; WOODGATE, R. L. Can I play? A concept analysis of participation in children with disabilities. Physical \& occupational therapy in pediatrics, v. 30, n. 4, p. 325-39, 2010. Disponível em: <http:// www.ncbi.nlm.nih.gov/pubmed/20735196>. Acesso em: 10 fev. 2013.

HWANG, A.-W.; et al. Applying the ICF-CY framework to examine biological and environmental factors in early childhood development. Journal of the Formosan Medical Association, , n. 17, p. 1-10, 2012. Elsevier Taiwan LLC. Disponível em: <http://linkinghub.elsevier.com/retrieve/pii/S0929664612000915>. Acesso em: 12 fev. 2013.

LAUDELINO, J. A. S.; NAVARRO, R. M.; BEUREN, I. B. Análise da abordagem da controladoria nas dissertaçōes e teses dos programas acadêmicos de mestrado e doutorado em ciências contábeis no Brasil. Revista de Contabilidade da UFBA, v. 4, n. 2, p. 21-33, 2010.

LAVILLE, C.; DIONNE, J. A construçáo do saber: manual de metodologia da pesquisa em ciências humanas. Porto Alegre: UFMG, 1999.

PIZANNI, L.; SILVA, R. C.; HAYASHI, M. C. P. I. Base de dados e bibliometria: a presença da Educaçấo Especial na base medline. Revista Brasileira de Biblioteconomia e Documentaçáo, v. 4, n. 1, p.68-85, 2008.

PIZZANI, L.; BELLO, S. F.; HAYASHI, M. C. P. I. Caracterização da produção científica em Educação Especial na Biblioteca Virtual em Saúde (BVS): um estudo bibliométrico. Revista Educaçáo Especial, Santa Maria, v.23, n.38, p. 379-398, set. /Dez.2010.

PLESS, M.; IBRAGIMOVA, N.; ADOLFSSON, M.; BJÖRCK-AKESSON, E.; GRANLUND, M. Evaluation of in-service training in using the ICF and ICF version for children and youth. Journal of rehabilitation medicine: official journal of the UEMS European Board of Physical and Rehabilitation Medicine, v. 41, n. 6, p. 451-8, 2009.

SAES, S. G. Estudo bibliométrico das publicaçôes em economia da Saúde, no Brasil, 1989-1998. (2000). 104p. Dissertação (Mestrado) - Faculdade de Saúde Pública, Universidade de São Paulo, São Paulo.

SILVA, M. R.; HAYASHI, C. R. M.; HAYASHI, M. C. P. I. Análise bibliométrica e cientométrica: desafios para especialistas que atuam no campo. CID: Revista de Ciência da Informaçáo e Documentaçáo. Ribeirăo Preto, v. 2, n. 1, p. 110-129, jan./jun. 2011.

SIMEONSSON, R. J.; et al. Students with disabilities: a national survey. Developmental and Behavioral Pediatrics, v. 423, p. 49-63, 2001.

SIMEONSSON, R. J.; LEONARD, M. ICF-CY: A universal tool for practice policy and research. Meeting of WHO collaboration centers for the family of international classifications. Tunis, Tunisia, v. 29, n. 4, 2006.

STÅHL, Y.; GRANLUND, M.; GÄRE-ANDERSSON, B.; ENSKÄR, K. Review Article: Mapping of children's health and development data on population level using the classification system ICF-CY. Scandinavian journal of public health, v. 39, n. 1, p. 51-57, 2011.

TOKUNAGA, A.; et al.; Kengo. Implementation and Future Strategies of ICF-CY for Special Needs Education (SNE) in Japan. Meeting of WHO collaboration centers for the family of international classifications. Trieste, Italy, v. 28, n. 3, 2007.

TRABACCA, A.; RUSSO, L.; LOSITO, L. et al. The ICF-CY perspective on the neurorehabilitation of cerebral palsy: a single case study. Journal of child neurology, v. 27, n. 2, p. 183-90, 2012. Disponível em: <http:// www.ncbi.nlm.nih.gov/pubmed/21911416>. Acesso em: 12 fev. 2013.

VIANNA, W. B.; ENSSLIN, L.; GIFFHORN, E. A integração sistêmica entre pós-graduação e educação básica no Brasil: contribuição teórica para um "estado da arte”. Ensaio: Avaliaçáo de Políticas Públicas em Educaçáo, v.19, n.71, p. 327-344, 2011

WHO World Health Organization. International Classification of Functioning, Disability and Health Children \& Youth Version. Geneva, 2007. 
Cristiane Paiva-Alves - Aline Cirelli Coppede - Maria Cristina Piumbato Innocentini Hayashi

- Cláudia Maria Simões Martinez

\section{Correspondência}

Cristiane Paiva Alves - Universidade Estadual Paulista Júlio de Mesquita Filho, Faculdade de Filosofia e Ciências - Campus de Marília. Rua Hygino Muzy Filho, 737, Mirante, CEP: 17525-000 - Marília, São Paulo - Brasil.

E-mail: crispaiva@marilia.unesp.br - alinecirelli@gmail.com -dmch@ufscar.br - claudia@ufscar.br

Recebido em 09 de março de 2015

Aprovado em 13 de abril de 2016 